\title{
Uma agenda para investigação em políticas e sistemas de saúde no Nordeste
}

\author{
A research agenda for health policies \\ and services in northeast Brazil
}

\footnotetext{
1 Instituto de Saúde Coletiva, Universidade Federal da Bahia. Rua Padre Feijó 29, 4o andar, Salvador, BA 40110-170, Brasil.
}

Abstract Theaim of this paper is to gather elements for a discussion on health policies and services in northeast Brazil. The author begins with an analysis of the population's main health problems, the health system's limitations, and current opportunities for implementing health programs. She highlights the foll owing research priorities: people's heal th situations according to their material living conditions and lifestyles; the private health sector's characteristics and devel opment; the current decentralization process; and proposed innovations in services, programs, and systems. The author points out further that the policy-making process in science and technology should assure a balance between researchers' freedom and peopl e's health needs, regional focus, and universal issues.

Key words Science; Technology; Research; Health Services

Resumo O presente artigo tem por objetivo reunir subsí di os para um debate sobre as prioridades na investigação em políticas e si stemas de saúde no Nordeste do Brasil. A partir de uma análise que leva em conta as macro-questões referentes ao estado de saúde da população desta re gião, os problemas do si stema de saúde e as oportuni dades presentes na atual conjuntura sanitária, são indi cadas as segui ntes áreas temáti cas como al gumas dentre aquel as rel evantes para a investigação: análi se de si tuações de saúde segundo condi ções e modos de vida; características e desenvolvimento do setor privado; dimensão e significados do atual processo de muni cipalização; desenvolvimento e avaliação de propostas inovadoras de model os assistenci ais; e avaliação de práticas, programas e sistemas de saúde. Destacou-se que o processo de formulação de políti cas indutoras de Ciência e Tecnol ogia em saúde corresponde a práti cas que requerem o equacionamento do conflito que se estabel ece entre a liberdade de criação dos i nvestigadores e os problemas rel aci onados com as necessidades de saúde da população, levando-se em conta as dimensões local, particular e universal do saber.

Palavras-chave Ciência; Tecnologia; Pesquisa; Serviços de Saúde 
Introdução

A adoção de uma política de Ciência eTecnologia capaz de conciliar a definição de agendas socialmente relevantes com a liberdade e a criatividade dos investigadores é uma tarefa complexa. Para sua viabilização, é indispensável a incorporação de propósitos e perspectivas dos diversos atores e instituições envolvidos, tanto os do campo científico, quanto os da saúde e os do poder.

Há uma constante tensão entre essas três dimensões, que podem ser contraditórias quando predomina o pragmatismo dos gestores ou um certo tipo de alienação do investigador. No primeiro caso, o Estado pode reduzir o apoio à pesquisa básica pelo fato de os seus resultados não terem visibilidade imediata. Por outro lado, quando o pesquisador se isola pode, por vezes, deixar de identificar problemas relevantes e originais de investigação que a vida cotidiana fornece. A necessidade da adoção de políticas indutoras de Ciência e Tecnologia, numa área como a de saúde, intimamente relacionada com os processos da vida, e, sobretudo com a qualidade de vida, vem sendo reconhecida por um número cada vez maior de pesquisadores, resguardado o espaço para a pesquisa básica.

Quanto à criação de consenso a esse respeito, nos diversos âmbitos, a exemplo da Conferência Nacional de Ciência e Tecnologia realizada em 1994, não é inviável nem contradiz o cuidado referente às armadilhas decorrentes dos objetos "pré-construídos" que, muitas vezes, colocam a pesquisa numa camisa-de-força (Bourdieu, 1989). Ou seja, uma agenda de investigação induzi da deve ser a mais ampla possível para que a construção do objeto de investigação não se desloque do campo científico para a esfera da técnica.

É importante enfatizar algumas considerações sobre as relações entre o regional e o universal na atividade de investigação. Esses dois âmbitos são por vezes situados como antagônicos, e, freqüentemente, o último adquire um estatuto de maior legitimidade científica em relação ao primeiro. Em conseqüência há a proposta de se atribuir a al gumas Universidades e Instituições de pesquisa uma "vocação regional" por tomarem a problemática local como objeto de investigação, ficando a cargo de outras o desenvolvimento de pesquisa de ponta. Nada mais equivocado. Se a pesquisa é de qualidade, ela vai revelar o universal a partir do local e do particular. Ou seja, para que a investigação científica possa contribuir na resolução dos problemas regionais ela tem que produzir conhecimento original, o que a torna necessariamente relevante e universal.

Por último, um comentário sobre a prática enquanto objeto da investigação científica. Particularmente na área de saúde, onde a pesquisa básica e a clínica são hegemônicas, é freqüente imaginar-se que o processo de produção de conhecimento científico esgota-se na produção de tecnologia. A aplicação e a operação das tecnologias passariam a ser, então, objeto situado fora do campo científico. No entanto, as práticas de saúde podem ser um objeto de investigação tão relevante quanto a biologia ou a imunologia. Por exemplo, o tratamento da tuberculose ou da hipertensão, agravos para os quais se dispõe de tecnologias de eficácia comprovada, requer a adoção de estratégias organizacionais e interpessoais capazes de assegurar a aderência dos sujeitos às prescrições e por conseguinte, à efetividade. Por outro lado, as formas pelas quais as ações sanitárias voltadas para o controle de danos, riscos e causas podem ser implementadas, requerem, em cada caso, desenvolvimento tecnológico relacionado com a gestão e a organização de serviços (Paim, 1993).

\section{Os problemas de saúde da Região}

Uma situação de saúde pode ser caracterizada a partir dos problemas, atores e do contexto social ao qual pertence. Os problemas podem ser tanto referentes ao estado de saúde da população quanto às práticas, serviços e sistemas. A definição de problema de saúde pode variar de acordo com o ponto de vista dos diversos atores sociais envolvidos. Por esse motivo, é que, em diversas técnicas de planificação, a obtenção de consenso entre técnicos, profissionais e usuários vem sendo privilegiada para a seleção e hierarquização de problemas (Matus, 1987). O debate sobre a relevância dos problemas de saúde de uma dada região pode ser considerado como movimento preliminar ao processo de definição de pautas prioritárias de investigação e pode ser iniciado a partir da ótica técnico-científica.

Nesse sentido, podem ser identificados dois macro-problemas na Região Nordeste: a polarização epidemiológica e as desigualdades na saúde. Constituindo-se em problema terminal, a polarização epidemiológica é resultante da coexistência, superposição e interação de processos produtores da denominada morbidade moderna (doenças cardiovasculares, neoplasias e violência) com a persistência, emergência e a reintrodução de doenças infecciosas 
(Barreto \& Carmo, 1994). Como os múltiplos fatores interagem na produção desse quadro? Quais os fenômenos relacionados com a sua evolução? Quais os nós críticos que permitem uma intervenção impactante? Quais as combinações tecnológicas capazes de modificá-lo? A resposta a essa última questão tem sido objeto de estudos que vão desde a Reforma Sanitária enquanto estratégia global de reorganização setorial até o desenvolvimento e avaliação de model os assistenciais alternativos (Paim, 1994).

O segundo macro problema selecionado as desigualdades em saúde - encontra-se intimamente relacionado ao primeiro. Esse é um bom exemplo de uma problemática que, numa primeira aproximação, revela um caráter regional mas que, na realidade, reflete um fenômeno universal e contemporâneo, identificado mesmo naqueles países possuidores de sistemas de saúde mais equânimes e de uma meIhor distribuição da riqueza social como Inglaterra (Fox \& Benzeval, 1995), Canadá (PampaIon, 1993) e Suécia (Westerling et al., 1996). O fenômeno vem sendo registrado em quase todos os países industrializados (Pappas et al., 1993; Kunst \& Mackenbach, 1994; Backlund et al., 1996), constituindo-se hoje em prioridade da Organização Mundial da Saúde (OMS), que vem desenvolvendo uma iniciativa global com o objetivo de promover e apoiar políticas e pesquisas voltadas para reduzir as diferenças sociais em saúde (WHO, 1996).

Na América Latina e no Brasil em particular, o fenômeno também tem sido estudado (Breilh et al., 1990; Paim \& Costa, 1993; Duncan et al., 1995; Vieira-da-Silva et al., 1999). A principal especificidade das desigualdades em saúde no Nordeste pode ser encontrada na sua magnitude. Enquanto que a mortalidade entre trabaIhadores braçais é de 2,5 a 3 vezes maior que aquela verificada entre profissionais liberais na Inglaterra (Fox \& Benzeval, 1995), em Salvador - a Zona de Informação corresponde ao bairro Sete de Abril, onde a maioria das famílias é menos favorecida econômica e culturalmente--, a mortalidade é 7,6 vezes maior que aquela observada no Itaigara, bairro em que a maioria das famílias tem maior acesso a bens econômicos e culturais (Vieira-da-Silva et al., 1999).

Qual a situação das desigualdades em saúde no Nordeste? Como têm evoluído? Quais os seus determinantes? Quais as suas conseqüências? A pertinência dessas questões, entre outras razões já mencionadas, relaciona-se com as limitações dos Sistemas de Informação em Saúde existentes, que ainda não produzem indicadores desagregados segundo condições de vida ou estratos sociais, como éfeito por exem- plo na Inglaterra desde 1923 (Fox \& Benzeval, 1995). Enquanto as estatísticas oficiais produzirem dados que homogeneízam artificialmente a realidade, a descrição da situação atual e da evolução das desigualdades se constituem em produção de conhecimento relevante. Por outro lado, existem lacunas relacionadas com as estratégias metodológicas e técnicas voltadas para a estratificação do espaço social no Brasil visando o monitoramento da situação de saúde.

\section{Problemas do sistema de saúde}

As políticas de saúde, posteriores à promulgação da Constituição de 1988, podem ser apreendidas e avaliadas através da análise das formas concretas de organização dos serviços de saúde no país. Nesse sentido, destacam-se quatro problemas principais: 1) Expansão anárquica do setor privado às custas das diversas modalidades do modelo assistencial privatista voltado para o atendimento à demanda espontânea. 2) Redução do projeto da reforma sanitária ao âmbito municipal, com conservação do modelo assistencial. 3) Persistência de assistência médica precária e discriminatória. 4) Efetividade e qualidade pouco conhecidas quando não ignoradas.

O setor privado de prestação de serviços na área da saúde tem-se expandindo no Brasil desde a década de 70 , inicialmente às custas do financiamento público, e, posteriormente, em decorrência das diversas formas de assistência médica supletiva (Mendes, 1993; Vieira-da-Silva et al., 1997). Essa expansão ocorreu de forma anárquica, sem levar em conta as reais necessidades da população, e, fundamentalmente centrada no atendimento à demanda espontânea. A complexidade e a natureza da determinação do quadro sanitário da Região, numa conjuntura de escassez de recursos, requer intervenção planificada, voltada, prioritariamente, para o controle de causas e riscos, o que envolve ações de saneamento e controle da poluição ambiental, modificações nos ambientes de trabalho, rotinas de comunicação social relacionadas com a difusão de estilos de vida saudáveis, práticas sociais organizadas para a redução da violência, etc. Em síntese, esse conjunto de ações requer intervenção do Estado e regulação do mesmo sobre o setor privado como ocorre em todos os sistemas de saúde que conseguiram enfrentar alguns dos problemas mencionados (Banoob, 1994).

Concomitantemente, verificou-se um retrocesso no projeto da Reforma Sanitária Brasileira que não conseguiu ampliar a eqüidade setorial através da organização de um sistema 
de saúde acessível, efetivo e de qualidade (Paim, 1996). A municipalização implementada, na maioria das vezes manteve o modelo assistencial anterior, bastante influenciado pela lógica da organização privada dos serviços quando não substituído pela mesma. Ou seja, descentralizou-se a gestão sem modificação nas formas de organização das práticas de saúde.

A despeito das dificuldades anteriormente mencionadas, dois fatos devem ser considerados: a existência de experiências inovadoras no âmbito municipal e estadual, mesmo que localizadas, e a implantação de programas especiais com potencial de impacto sobre alguns problemas, a exemplo do Programa de Saúde da Família e do Programa dos Agentes Comunitários de Saúde. O VIGISUS, projeto em elaboração no Ministério da Saúde para a montagem de um sistema nacional de vigilância à saú de com o objetivo de mudança do modelo assistencial, é ainda uma promessa; porém, caso se concretize, oportunizará o desenvolvimento de investigações operacionais.

Diante dos problemas acima identificados bem como das oportunidades consideradas, pode-se pensar numa pauta de prioridades para a investigação e solução dos mesmos: 1) Análise de situações de saúde segundo condições, estilos e modos de vida; 2 ) Características e desenvolvimento do setor privado - relações público x privado; 3) Dimensão e significados do atual processo de municipalização; 4) Desenvolvimento e avaliação de propostas inovado-

\section{Agradecimentos}

A autora agradece os comentários e sugestões de Jairnilson Paim e Naomar de Almeida Filho. O trabal ho contou com o apoio financeiro do Consel ho Nacional de Desenvolvimento Científico e Tecnológico (CNPq). res de modelos assistenciais; 5) Avaliação de práticas, programas e sistemas de saúde: eficácia, efetividade, eficiência, qualidade, satisfação/percepção dos usuários, acessibilidade, eqüidade, cobertura e análise de implantação de programas.

\section{Considerações finais}

Para finalizar, o sucesso de uma política científica dessa natureza está na parceria que as instituições de saúde devem estabelecer com o poder político, administrativo e técnico. A adoção de práticas simples como a vinculação dos bancos de dados de mortalidade e censos, adotada em diversos países, bem como a produção de indicadores desagregados segundo condições de vida ou estratos, são apenas alguns exemplos de iniciativas administrativas que já poderiam ter si do tomadas e que não só potencializariam as investigações sobre algumas das temáticas discutidas, como poderiam fornecer informações relevantes para a intervenção sanitária. Sabemos que a realização de investigação voltada para a resolução dos problemas de saúde da população, a seleção de tecnologias e a conseqüente tomada de decisões com respaldo científico, muitas vezes contrariam interesses pessoais, de grupos e de classes. Assim, a solução do problema se desloca de campo e a bola passa para os governantes. Cabe-nos, porém, fazer o passe bem feito.

\section{Referências}

BACKLUND, E.; SORLIE, P. D. \& JOHNSON, N. J. 1996. The shape of the relationship between income of Epidemiology and mortality in the United States. Evidence from the national longitudinal mortality study. Annals of Epidemiology, 6:12-20.

BANOOB, S., 1994. Réforme sanitaire en Europe centrale et orientale: Financement public ou privé? Forum Mondial dela Santé, 15:351-357.

BARRETO, M. L. \& CARM O, E. H., 1994. Situação de saúde da população brasileira: Tendências históricas, determinantes e implicações para as políticas de saúde. Informe Epidemiológico do SUS, 3:7-34. 
BOURDIEU, P., 1989. O Poder Simbólico. Lisboa: DIFEL/Rio de Janeiro: Editora Bertrand Brasil.

BREILH, J. G. E.; CAM PAÑA, A.; YEPEZ, J.; PAEZ, R. \& COSTALES, P., 1990. Deterioro de la Vida. Un Instrumento para Análisis de Prioridades Regionales en Io Social y la Salud. Quito: Corporación Editora Nacional.

FOX, J. \& BENZEVAL, M., 1995. Perspectives on social variations in health. In: Tackling Inequalities in Health. An Agenda for Action (M. Benzeval, K. Judge \& M. Whitehead, eds.), pp. 10-21, London: King's Fund.

KUNST, A. E. \& MACKENBACH, J., 1994. The size of mortality differences associated with educational level in nine industrialized countries. American Journal of Public Health, 84:932-937.

MATUS, C., 1987. Política, Planificación y Gobierno. Washington, D.C.: Organización Panamericana de la Salud/Instituto Latino Americano y del Caribe de Planificación Económica y Social.

MENDES, E. V. R. E. A., 1993. Distrito Sanitário. O Processo Social de Mudanças das Práticas Sanitárias do Sistema Único de Saúde. São Paulo: Editora Hucitec/ Rio de Janeiro: ABRASCO.

PAIM , J. S., 1993. A reorganização das práticas em distritos sanitários. In: Distrito Sanitário. O Processo Social de Mudança das Práticas Sanitárias do Sistema Ú nico deSaúde(E. V. Mendes, org.), pp. 187220, São Paulo: Editora Hucitec/Rio de Janeiro: ABRASCO.

PAIM , J. S., 1994. A reforma sanitária e os modelos assistenciais. In: SaúdeColetiva. Textos Didáticos (L. M. Vieira-da-Silva, org.), pp. 61-82, Salvador: Centro Editorial e Didático, Universidade Federal da Bahia.
PAIM, J. S., 1996. O SUS no ensino médico: Retórica ou realidade? Divulgação em Saúde Para Debate, 14:59.

PAIM, J. S. \& COSTA, M. C. N., 1993. Decréscimo e desigualdade da mortalidade infantil, Salvador, 1980-1988. Boletín dela Oficina Sanitaria Panamericana, 114:415-428.

PAM PALON, R., 1993. Avoidable mortality in Québec and its regions. Social Science and Medicine, 37: 823-831.

PAPPAS, G.; QUEEN, S.; HADDEN, W. \& FISHER, G., 1993. The increasing disparity in mortality between socioeconomic groups in the United States, 1960-1986. New England Journal of Medicine, 329:103-109.

VIEIRA-DA-SILVA, L. M.; PAIM, J. S. \& COSTA, M. C. N., 1999. Desiqualdades na mortalidade, espaço e estratos sociais em uma capital da região nordeste. Revista deSaúde Pública de São Paulo, 33:18797.

VIEIRA-DA-SILVA, L. M.; SOUZA, L. E. P. F.; CERDEIRA, A. J. P.; PINTO, C. M. F. \& OLIVEIRA, R. S. A., 1997. Algumas características do setor privado de saúde de Salvador. Cadernos de Saúde Pública, 13:701-709.

WESTERLING, R.; GULBERG, A. \& ROSEN, A. M., 1996. Socioeconomic differences in avoidable mortality in Sweden 1986-1990. International Journal of Epidemiology, 25:560-567.

WHO (World Health Organization), 1996. Equity in Health and Health Care: A WHO/SIDA Iniciative. Geneva: WHO. 